\title{
Correction to: Genetic and morphological sex identification methods reveal a male-biased sex ratio in the Ivory Gull Pagophila eburnea
}

\author{
Glenn Yannic ${ }^{1,2} \cdot$ Thomas Broquet $^{3,4} \cdot$ Hallvard Strøm $^{5}$. Adrian Aebischer ${ }^{2} \cdot$ Christophe Dufresnes $^{6}$. \\ Maria V. Gavrilo ${ }^{7,8} \cdot$ H. Grant Gilchrist ${ }^{9} \cdot$ Mark L. Mallory $^{10} \cdot$ R. I. Guy Morrison ${ }^{11} \cdot$ Brigitte Sabard $^{2} \cdot$ Roberto Sermier $^{6}$. \\ Olivier Gilg ${ }^{2,12}$
}

Published online: 9 March 2018

(c) Dt. Ornithologen-Gesellschaft e.V. 2018

Correction to: J Ornithol (2016) 157:861-873

https://doi.org/10.1007/s10336-016-1328-4

In the original publication of the article, the linear discriminate equation (1) was mistyped. We provide below the correct equation to use to discriminate male and female in Ivory Gull Pagophila eburnea (Phipps, 1774).

$$
\begin{aligned}
D= & -38.435+0.005 \times V_{\text {Weight }}+0.268 \times V_{\text {Wing }} \\
& +0.136 \times V_{\text {Skull }}+1.203 \times V_{\text {Gonys }} .
\end{aligned}
$$

According to this equation, a bird is classified as male when $D>0$ and as female when $D<0$.

The original article can be found online at https://doi.org/10.1007/ s10336-016-1328-4.

Glenn Yannic

glenn.yannic@gmail.com

1 LECA - Laboratoire d'Ecologie Alpine - CNRS UMR 5553, Université Savoie Mont Blanc, 73376 Le Bourget-Du-Lac Cedex, France

2 Groupe de Recherche en Ecologie Arctique (GREA), 21440 Francheville, France

3 CNRS, Team Diversity and Connectivity of Coastal Marine Landscapes, Station Biologique de Roscoff, 29680 Roscoff, France

4 UMR 7144, Station Biologique de Roscoff, Sorbonne Universités, UPMC Univ Paris 06, 29680 Roscoff, France

5 Norwegian Polar Institute, Polar Environmental Centre, 9296 Tromsö, Norway

6 Department of Ecology and Evolution, University of Lausanne, 1015 Lausanne, Switzerland
7 National Park Russian Arctic, 57 Sovetskikh Kosmonavtov pr., Archangelsk, Russia 163000

8 Joint Directorate of Taimyr Nature Reserves, 22 Talnakhskaya, Norilsk, Russia 663300

9 Environment Canada, National Wildlife Research Centre and Department of Biology, Carleton University, Ottawa, ON K1S5B6, Canada

10 Acadia University, 33 Westwood Avenue, Wolfville, NS B4P 2R6, Canada

11 Environment Canada, Science and Technology Branch, National Wildlife Research Centre, Carleton University, 1125 Colonel By Drive (Raven Road), Ottawa, ON K1A OH3, Canada

12 Laboratoire Biogéosciences, UMR CNRS 6282, Equipe Ecologie Evolutive, Université de Bourgogne, Boulevard Gabriel, 21000 Dijon, France 